\title{
A strong electromechanically coupled and low- damped harvester for resonant frequency tuning
}

\author{
David Gibus \\ Université Savoie Mont Blanc, SYMME \\ Annecy, France \\ david.gibus@univ-smb.fr \\ Adrien Ameye \\ Université Grenoble Alpes, CEA LETI \\ Grenoble, France
}

\author{
Pierre Gasnier \\ Université Grenoble Alpes, CEA LETI \\ Grenoble, France
}

\author{
Adrien Morel \\ Université Savoie Mont Blanc SYMME \\ Annecy, France
}

Adrien Badel

Université Savoie Mont Blanc SYMME

Annecy, France

\begin{abstract}
The present work introduces the design and fabrication of a strongly coupled piezoelectric vibration energy harvester dedicated to resonant frequency tuning by electrical methods. The electromechanical coupling coefficient of the harvester is maximized thanks to an analytical model and 3D FEM simulations in order to extend the frequency tuning bandwidth. Moreover, losses of candidate substrates are analyzed in order to maximize the mechanical quality factor of the harvester. A PZN-5.5PT and aluminum based cantilever is proposed and experimentally validated with resistive output loads. Among state-of-the-art solutions, the proposed prototype exhibits one of the best coupling coefficient $\left(k^{2}=41 \%\right)$ and normalized power density $\left(115 \mathrm{~kg} . \mathrm{s}^{\mathrm{m}} \mathrm{m}^{-3}\right)$.
\end{abstract}

$\begin{aligned} & \text { Keywords-Vibration Energy Harvesting, } \\ & \text { Frequency Tuning, Strong Electromechanical } \\ & \text { coefficient }\end{aligned}$

\section{INTRODUCTION}

Vibration energy harvesting is a relevant solution to supply energy to sensors where batteries cannot be changed easily [1]. Nevertheless, the frequency bandwidth is still a major issue for large scale industrialization of vibration energy harvesters. One up-rising solution is the use of power management circuits able to tune the resonant frequency of piezoelectric harvester. Such circuits based on synchronous charge extraction (SECE) and synchronized switch harvesting on inductor (SSHI) have indeed shown interesting performances on strongly coupled harvesters since the impact of the circuit on the harvester dynamics is maximized [2]. Nevertheless, in the literature, the electromechanical coupling coefficient of most harvesters remain relatively weak $\left(k^{2}<20 \%\right)$ [3], and no design guidelines are given for very strongly coupled harvester [4].

The present paper therefore introduces a design method for strongly coupled cantilevers using piezoelectric materials with very strong electromechanical coupling coefficients. The second section introduces theoretical guidelines based on an analytical expression of the global coupling coefficient of a piezoelectric cantilever. The third section discusses the specific optimization of such a cantilever conducted thanks to losses measurements and 3D FEM simulations. The last section is dedicated to the presentation of the high performance harvester and the experimental measurements.

\section{MODELING}

\section{A. Device configuration}

The harvester design relies on a piezoelectric bimorph with a long proof mass (Fig. 1) as it has been shown relevant to maximize the global electromechanical coupling coefficient [3]. The long proof mass is used in order to homogenize the longitudinal strain distribution in the beam and increases the global electromechanical coupling coefficient $k^{2}$.

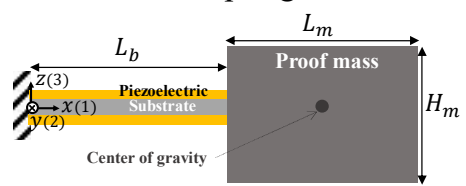

Fig. 1: Cantilever configuration. The width $B$ is in the out-of-plane direction.

The two piezoelectric patches are the same length as the substrate. They have the same thicknesses $\left(h_{p}\right)$ and are completely covered with electrodes on the bottom and top sides. The thickness of the substrate is given by $h_{s}$. The assumption of plane stress is considered because the beam is narrow (i.e. $L_{b}>3 B$ ). The proof mass is modelled by an equivalent point mass defined by a mass $M_{t}$ and a rotational inertia $I_{t}$ at a distance $D_{t}$ from the end of the beam on the neutral axis.

\section{B. Modelling}

In a previous work [3], we have shown that the electromechanical coupling of the first bending mode of a piezoelectric bimorph with proof mass can be expressed thanks to (1). $k_{e}{ }^{2}$ is the alternative coupling coefficient and is defined as $k_{e}^{2}=k^{2} /\left(1-k^{2}\right)$. In the same manner, $k_{e 31}{ }^{2}$ is the expedient coupling coefficient of the piezoelectric material according to the 31-mode: $k_{e 31}{ }^{2}=k_{31}{ }^{2} /\left(1-k_{31}{ }^{2}\right)$.

$$
k_{e}^{2}=k_{e 31^{2}} R_{L} R_{T}
$$

$R_{L}$ and $R_{T}$ are called length and thickness factors respectively. $R_{T}$ is related to the stress distribution in the thickness of the beam and is expressed in (2). $Y_{s}^{e f}$ is the Young modulus of the substrate, $c_{11} e^{e f}$ is the linear elastic coefficient of the piezoelectric material according to the plane stress assumption. As shown in (3), $R_{L}$ only depends on the strain distribution along the beam given by the $b / a$ ratio expressed in (4) with $\Gamma$ a coefficient expressed in (5). $J_{t}$ is the rotary inertia to mass ratio expressed as $J_{t}=I_{t} / M_{t}$.

$$
\begin{gathered}
R_{T}=\frac{\left(\frac{h_{p}}{h_{s}}\right)^{3}+2\left(\frac{h_{p}}{h_{s}}\right)^{2}+\left(\frac{h_{p}}{h_{s}}\right)}{\frac{1}{6}\left(\frac{Y_{s}^{e f}}{c_{11}^{e f}}\right)+\frac{4+k_{e_{31}}^{2}}{3}\left(\frac{h_{p}}{h_{s}}\right)^{3}+2\left(\frac{h_{p}}{h_{s}}\right)^{2}+\left(\frac{h_{p}}{h_{s}}\right)} \\
R_{L}=\frac{\left(\frac{b}{a}\right)^{2}+\left(\frac{b}{a}\right)+\frac{1}{4}}{\left(\frac{b}{a}\right)^{2}+\left(\frac{b}{a}\right)+\frac{1}{3}}
\end{gathered}
$$




$$
\begin{gathered}
\frac{b}{a}=-\frac{3 J_{t}-\Gamma^{\frac{1}{2}}+3 D_{t}^{2}+L_{b}^{2}+4 D_{t} L_{b}}{6 J_{t}-2 \Gamma^{\frac{1}{2}}+6 D_{t}^{2}+L_{b}^{2}+6 D_{t} L_{b}} \\
\Gamma=9 D_{t}^{4}+18 D_{t}^{3} L_{b}+15 D_{t}^{2} L_{b}^{2}+18 D_{t}^{2} J_{t}+ \\
6 D_{t} L_{b}^{3}+18 D_{t} L_{b} J_{t}+L_{b}^{4}+3 L_{b}^{2} J_{t}+9 J_{t}^{2}
\end{gathered}
$$

$R_{L}$ and $R_{T}$ are both smaller than one and the aim of the optimization process is to make them as close to unity as possible. The process of optimizing theoretically the longitudinal stress distribution and the transverse stress distribution (i.e. optimizing $R_{L}$ and $R_{T}$ ) can be carried out independently as they do not rely on the same parameters.

The optimization of $R_{L}$ has already been discussed in the previous paper [3]. The present work mainly focusses on the $R_{T}$ optimization with the consideration of very strongly coupled piezoelectric materials (i.e. $k_{31}{ }^{2}>15 \%$ ).

\section{Disscusion on $R_{T}$}

For given substrate and piezoelectric materials, $R_{T}$ is maximized for an optimal thickness ratio $\left(h_{p} / h_{s}\right)^{\text {opt }}$. This optimal ratio is determined by calculating the zero of the derivative of the $R_{T}$ expression and is given in (6). $\left(h_{p} / h_{s}\right)^{\text {opt }}$ depends only on a rigidity ratio $\kappa$ that is defined in (7).

$$
\begin{gathered}
\left(\frac{h_{p}}{h_{s}}\right)^{\text {opt }}=\left\{\begin{array}{lc}
\frac{1}{2} \kappa^{\frac{1}{3}}\left[(1-\sqrt{1-\kappa})^{\frac{1}{3}}+(1+\sqrt{1-\kappa})^{\frac{1}{3}}\right] & \text { for } \kappa \leq 1 \\
\sqrt{\kappa} \cos \left(\frac{\arctan \sqrt{\kappa-1}}{3}\right) & \text { for } \kappa>1
\end{array}\right. \\
\kappa=\frac{Y_{s}^{e f}}{c_{11}^{e f}} \frac{1}{k_{e_{31}}^{2}+1}
\end{gathered}
$$

The maximum achievable value of $R_{T}$ for given piezoelectric and substrate materials, noted $R_{T}^{\max }$, is determined by replacing $h_{p} / h_{s}$ in (2) by (6). Fig. 2 shows $R_{T}^{\max }$ as a function of the $\kappa$ ratio for three values of material coupling coefficient $\mathrm{k}_{31}{ }^{2}(15 \%, 25 \%$ and $81 \%)$. As $k_{31}$ is fixed for each curve, Fig. 2 amounts to analyzing the effect of the ratio of material stiffness $\left(c_{11}{ }^{e f}\right.$ and $\left.Y_{S}^{e f}\right)$ on $R_{T}^{\max }$. From this figure, we notice that, the stronger the coupling coefficient of the material, the more the value of the ratio $\kappa$ has an impact on the value of $R_{T}$ and, by extension, on the overall achievable coupling coefficient. Therefore, it seems challenging to make piezoelectric cantilevers having the same coupling coefficient as the piezoelectric material with very strongly coupled materials. Moreover, Fig. 2 reveals that the optimization of $\kappa$ through the choice of materials is therefore of paramount importance in the optimization of piezoelectric beams.

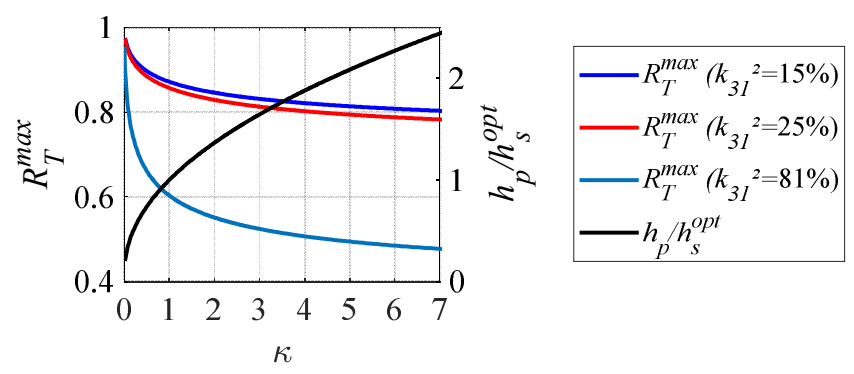

Fig. 2: Values of the optimal thickness ratio and the maximal value of $R_{T}$ as a function of the rigidity ratio $\kappa$
As this work is dedicated to materials with very strong coupling coefficient, the PZN-5.5PT [011] material have been chosen as a case of study. It is indeed one of the most coupled piezoelectric material regarding the 31 mode $\left(k_{31}^{2}=81 \%\right)$.

TABLE 1 contains the value of $\kappa$, the value of $R_{T}{ }^{\max }$ and the maximum achievable value for the coupling coefficient $k_{\max }{ }^{2}$ for combinations of PZN-PT and substrates (steel, aluminum and brass). The maximum coupling coefficient $k_{\max }{ }^{2}$ is calculated considering the longitudinal stress distribution as completely uniform $\left(R_{L}=1\right)$. Through these calculations, the maximum value of overall coupling coefficient achievable with a combination of piezoelectric material and substrate when the beam is considered in plane stress is determined.

TABLE 1: Values of $R_{T}^{\max }$. and $k_{\max }{ }^{2}$ for PZN-5.5PT [011] $\left(c_{11}{ }^{e f}=7.3 \mathrm{GPa}\right)$

\begin{tabular}{|c|c|c|c|c|}
\hline & $Y_{s}$ & $\kappa$ & $R_{T}^{\max }$ & $k_{\max }^{2}$ \\
\hline Aluminium & $69 \mathrm{GPa}$ & 1.8 & 0.56 & $70 \%$ \\
\hline Brass & $110 \mathrm{GPa}$ & 2.9 & 0.53 & $69 \%$ \\
\hline Steel & $200 \mathrm{GPa}$ & 5.2 & 0.49 & $68 \%$ \\
\hline
\end{tabular}

It can be deduced from TABLE 1 that the material used as a substrate has a influence on the maximum coupling coefficient. As an example, the coupling coefficient of a beam comprising PZN-PT and steel cannot exceed $68 \%$ while the material coupling $k_{31}{ }^{2}$ of PZN-PT is $81 \%$.

This previous theoretical analysis showed that the design of a cantilever is affected by the materials choice. To go further, we show in the next section, with the example of the design of a strongly coupled harvester, that the design choices must also take material losses and the configuration of the prototype into account.

\section{DESIGN CONFIGURATION}

\section{A. Design constraint}

While coupling coefficient is an important criterion for the frequency bandwidth, the mechanical quality factor of the harvester has also to be maximized in order to increase the harvested power. Considering that the air damping is negligible compared to the material losses in mesoscale harvesters, the overall losses of a harvester are strongly dependents on the structural losses of the materials.

In addition, the manufacturing configuration of the harvester can lead to strategic dimensioning choices. Indeed, due to the configuration of the prototypes, spacings are required between the patches and the stationary frame at one end and between the patches and the proof mass at the opposite end. These spacings are noted $d_{c}$ and $d_{m}$ respectively in Fig. 3 Although these distances and the thickness of the adhesive between the patches and the substrate cannot be determined precisely and are limited, they induce a drastic decrease in $k^{2}$. The influence of the spacings can be analyzed through a 3D finite element simulation software.

This section considers both of these aspects (the material losses and the spacings) simultaneously to finalize the design of a strongly coupled harvester. 


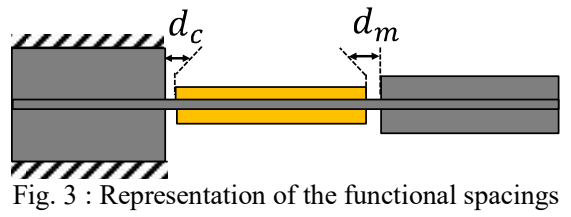

\section{B. Design constraint}

The aim of our work is to design and fabricate a harvester with small dimensions and a short-circuit resonant frequency of less than $200 \mathrm{~Hz}$. Metals (brass, steel and aluminum) were considered for the substrate materials as they have the advantage of having low loss factors and being easily machined by mechanical parts suppliers. The proof mass is made of steel thanks to its availability and its high density.

Each substrate material (brass, steel and aluminum) was considered to provide a prototype configuration. The material that provides the best coupling coefficient, despite the spacings, in combination with the lowest loss factor is selected. The length of the beam $L_{b}$ and the proof mass shape have been optimized to maximize the $R_{L}$ factor and adjust the resonant frequency in a restrained volume. Furthermore, several geometric parameters have been established due to production constraints of the suppliers. The shared geometric parameters established for the three substrate materials are shown in TABLE 2 .

TABLE 2: Geometrical parameters considered for the design
\begin{tabular}{|l|l|}
\hline Beam length $\mathrm{Lb}$ & $15 \mathrm{~mm}$ \\
\hline Proof mass length $\mathrm{Lm}$ & $20 \mathrm{~mm}$ \\
\hline Proof mass heigth $\mathrm{Hm}$ & $4 \mathrm{~mm}$ \\
\hline Patches thickness $\mathrm{hp}$ & $0.4 \mathrm{~mm}$ \\
\hline Beam and proof mass width & $5 \mathrm{~mm}$ \\
\hline
\end{tabular}

The expected coupling coefficients of the harvesters for the optimal substrate thicknesses were calculated thanks to 3D modal analyses on Comsol Multiphysics (Fig. 4) at shortcircuit and open-circuit conditions. These calculations were conducted considering a perfect bonding as well as with $\mathrm{d}_{\mathrm{c}}$ and $\mathrm{d}_{\mathrm{m}}$ spacings equal to $50 \mu \mathrm{m}$. In addition, the loss factors $\eta$ of the three metals were measured by vibration tests $\left(1 \mathrm{~m} / \mathrm{s}^{2}\right.$ acceleration amplitude) on bare beams of the substrate size. Based on the supplier datasheet [5], the quality factor $Q_{m}$ of $\mathrm{PZN}-5.5 \mathrm{PT}$ [011] is equal to 80 (i.e mechanical loss factor $\eta=1 / Q_{m}$ equal to 0.0125$)$. The overall expected loss factor of the harvester could therefore be deduced by the model. The results are resumed in TABLE 3 .
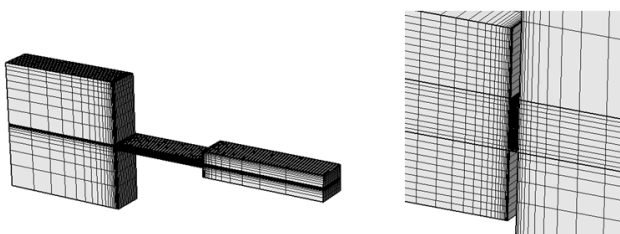

Fig. 4: Meshing on Comsol Multiphysics for the functional clearance

TABLE 3: Substrate thicknesses, short-circuit resonant frequencies $f_{s c}$ and coupling coefficients $k^{2}$ obtained with 3D Comsol Multiphysics. Measured loss factors $\eta$ of the metals and overall expected values for the harvesters.

\begin{tabular}{|c|c|c|c|c|c|c|}
\hline \multirow{2}{*}{ Material } & $\begin{array}{c}\text { Optimal } \\
\text { substrate } \\
\text { thickness } \\
\end{array}$ & \multicolumn{2}{|c|}{$\begin{array}{c}\text { Perfect } \\
\text { clamping }\end{array}$} & $\begin{array}{c}\text { Spacing } \\
h_{c}=d_{m}= \\
50 \mu \mathrm{m}\end{array}$ & $\begin{array}{c}\text { Measured } \\
\eta \text { of metal }\end{array}$ & $\begin{array}{c}\text { Overall } \\
\text { expected } \\
\text { loss factor }\end{array}$ \\
\cline { 3 - 7 } & $f_{s c}$ & $k^{2}$ & $k^{2}$ & & \\
\hline Aluminum & $0.5 \mathrm{~mm}$ & $130 \mathrm{~Hz}$ & $60 \%$ & $54 \%$ & 0.004 & 0.004 \\
\hline Brass & $0.4 \mathrm{~mm}$ & $118 \mathrm{~Hz}$ & $59 \%$ & $53 \%$ & 0.003 & 0.004 \\
\hline Steel & $0.3 \mathrm{~mm}$ & $108 \mathrm{~Hz}$ & $57 \%$ & $51 \%$ & 0.002 & 0.004 \\
\hline
\end{tabular}

According to TABLE 3, the spacings induces drastic decrease of the global coupling coefficient. The three design propositions offer close resonant frequencies and the aluminum configuration offers the best coupling coefficient when the spacings are considered $\left(k^{2}=54 \%\right)$.

The steel substrate offers the lowest lost factor of the three metals. However, since the loss factor of PZN-PT is higher than the metals loss factors, a higher proportion of substrate to PZN-PT leads to lower losses in the overall prototype. Because of the higher proportion of substrate to PZN-PT in the aluminum prototype, the equivalent quality factor is expected to be almost the same than with brass or steel. Therefore, the material aluminum and the given dimensions were chosen as the substrate for the prototype.

\section{MEASUREMENTS AND RESULTS}

\section{A. Fabrication and experiments}

The PZN-PT patches were cut at size by the manufacturer Microfine and bonded with epoxy glue in our laboratory to the aluminum beam. The proof mass was also bonded to the substrates with an epoxy adhesive. Fig. 5 depicts the final prototype.

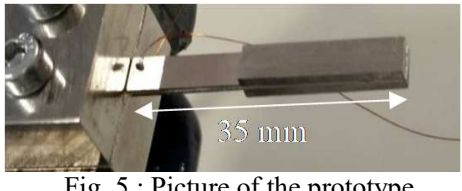

Fig. 5 : Picture of the prototype

The power output of the prototype was determined under vibration excitation (Fig. 6). The vibrations are generated and controlled by an electromagnetic vibrator (K2075E-HT) driven by a DSpace board and the acceleration level is monitored using an accelerometer (Piezotronics 356A17 PCB). The DSpace board is controlled by a dedicated Matlab script that defines the level, the acceleration frequency and controls the programmable electrical resistance. Experiments were performed for 60 resistive loads logarithmically spaced between $500 \Omega$ and $20 \mathrm{M} \Omega$ over 400 excitation frequencies between $105 \mathrm{~Hz}$ and $155 \mathrm{~Hz}$.

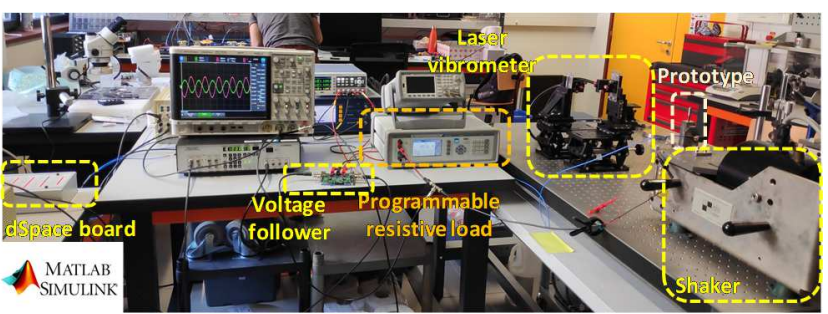

Fig. 6 : Experimental test bench

The harvested mean power is represented in Fig. 7. As the prototype exhibits a softening behavior and nonlinear losses with the variation of the acceleration level, the measurements have been fitted with the one mechanical degree of freedom nonlinear model presented in [6]. We notice that the results deduced from the model are close to the measurements despite the wide frequency range of measurements. 

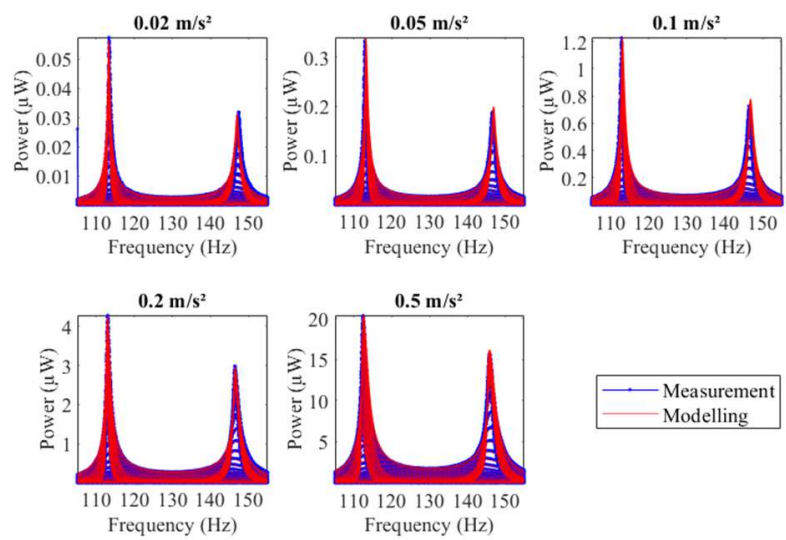

Fig. 7 : Experimental power

\section{B. Coupling coefficient and bandwidth}

Two power peaks are present in the plots in Fig. 7 as the prototype exhibits a strong coupling coefficient and a high quality factor. The global coupling coefficient is therefore estimated thanks to the frequency at which power peaks are reached at low acceleration level. These frequencies are indeed close to the short-circuit resonant frequency $f_{s c}$ and to the open-circuit resonant frequency $f_{o c}$. The global coupling coefficient can be computed by $k^{2}=\left(f_{o c}{ }^{2}-f_{s c}{ }^{2}\right) / f_{o c}{ }^{2}$ and is equal to $k^{2}=41 \%$. Although this value does not exactly equal the simulation results due to large uncertainties in spacing and glue thickness, the present harvester is one of the most coupled harvesters in the state of the art.

As explained in [6], [7], the power can be maximized with optimal capacitor and resistor pairs or some electrical techniques (e.g. SC-SECE [2]) in the frequency range between the power peaks given by resistors. With this assumption, the proposed harvester could offer a range of tunability between the peaks and the expected relative bandwidth of the harvester at the half of the maximal power would be equal to $27 \%$ (between $112 \mathrm{~Hz}$ and $147 \mathrm{~Hz}$ ) at the acceleration amplitude of $0.5 \mathrm{~m} / \mathrm{s}^{2}$.

\section{Quality factor and Normalized Power Density}

Thanks to the model, we know that the linear quality factor at $0.5 \mathrm{~m} / \mathrm{s}^{2}$ is equal to around 150 at the short-circuit resonant frequency (equivalent loss factor equal to 0.007). This result has been made possible thanks to the choice of material and the limitation of the strain amplitude in the piezoelectric material (to avoid strong nonlinear losses). The proposed harvester offers sufficient power to supply a wireless sensor node $\left(20 \mu \mathrm{W}\right.$ at $\left.0.5 \mathrm{~m} / \mathrm{s}^{2}\right)$ in a small overall volume. As an illustration, the normalized power density (NPD) given by $\mathrm{NPD}=$ Power $/\left(\right.$ Overall Volume $\times$ Acceleration $\left.^{2}\right)$, is equal to $115 \mathrm{~kg} . \mathrm{s} . \mathrm{m}^{-3}$ at $0.5 \mathrm{~m} / \mathrm{s}^{2}$ acceleration. This is one of the best NPD in the state of the art of strongly coupled harvesters as shown in TABLE 4.

TABLE 4: State-of-the-art of strongly coupled harvesters

\begin{tabular}{|c|c|c|c|c|}
\hline Ref. & {$[8]$} & {$[3]$} & {$[2]$} & This work \\
\hline Material & PZN-PT & PMN-PT & PZN-PT & PZN-PT \\
\hline Coupling & $50 \%$ & $17 \%$ & $32 \%$ & $41 \%$ \\
\hline Volume & $6750 \mathrm{~mm}^{3}$ & $4500 \mathrm{~mm}^{3}$ & $1000 \mathrm{~mm}^{3}$ & $700 \mathrm{~mm}^{3}$ \\
\hline Acceleration & $1.4 \mathrm{~m} / \mathrm{s}^{2}$ & $0.5 \mathrm{~m} / \mathrm{s}^{2}$ & $0.8 \mathrm{~m} / \mathrm{s}^{2}$ & $0.5 \mathrm{~m} / \mathrm{s}^{2}$ \\
\hline Mean Power & $480 \mu \mathrm{W}$ & $163 \mu \mathrm{W}$ & $35 \mu \mathrm{W}$ & $20 \mu \mathrm{W}$ \\
\hline Bandwidth & $32.7 \%$ & $12.3 \%$ & $43.5 \%$ & $27 \%$ \\
\hline NPD & $36.9 \mathrm{~kg}$. s.m $^{-3}$ & $146 \mathrm{~kg} . \mathrm{s}^{-3}$ & $48 \mathrm{~kg} . \mathrm{s}^{-3} \mathrm{~m}^{-3}$ & $115 \mathrm{~kg} . \mathrm{s}^{-3}$ \\
\hline
\end{tabular}

\section{CONCLUSION}

This work presents the design of strongly coupled harvester dedicated to the resonant frequency tuning thanks to electrical interfaces. An analytical expression of the electromechanical coupling coefficient of piezoelectric cantilevers has been firstly introduced to provide general optimization guidelines. As an illustration, soft substrate material should be preferred for maximizing the global electromechanical coupling coefficient of harvester. Nevertheless, intrinsic losses of the materials and the presence of spacings and manufacturing variability have also to be considered to design harvester. This have been done in this work thanks to loss measurement on substrates beam and 3D Comsol simulations respectively. A strongly coupled cantilever realized with PZN-5.5PT [011] and aluminum is finally proposed. The prototype has been tested under harmonic vibration excitation with output resistive loads. The harvester produces $20 \mu \mathrm{W}$ at $0.5 \mathrm{~m} / \mathrm{s}^{2}$ acceleration amplitude. It is expected to offer a $27 \%$ relative bandwidth when associated with pairs of optimal capacitive and resistive loads and have a normalized power density of $115 \mathrm{~kg} .{\mathrm{s} . \mathrm{m}^{-3}}^{-3}$ Experiments will be conducted with dedicated nonlinear electrical interface (e.g. SC-SECE).

\section{REFERENCES}

[1] S. Priya et al., "A Review on Piezoelectric Energy Harvesting: Materials, Methods, and Circuits," Energy Harvest. Syst., vol. 4, no. 1, Art. no. 1, Aug. 2019, doi: 10.1515/ehs-2016-0028.

[2] A. Morel, P. Gasnier, Y. Wanderoild, G. Pillonnet, and A. Badel, "Short Circuit Synchronous Electric Charge Extraction (SC-SECE) Strategy for Wideband Vibration Energy Harvesting," IEEE International Symposium of Circuits And Systems (ISCAS), 2018.

[3] D. Gibus et al., "Strongly coupled piezoelectric cantilevers for broadband vibration energy harvesting," Appl. Energy, vol. 277, p. 115518, Nov. 2020, doi: 10.1016/j.apenergy.2020.115518.

[4] A. Badel and E. Lefeuvre, "Wideband Piezoelectric Energy Harvester Tuned Through its Electronic Interface Circuit," J. Phys. Conf. Ser., vol. 557, p. 012115, Nov. 2014, doi: 10.1088/17426596/557/1/012115.

[5] “" Microfine," Apr. 30, 2020. https://microfinepiezo.com/product.php?id=1 (accessed Apr. 30, 2020).

[6] D. Gibus, P. Gasnier, A. Morel, N. Garraud, and A. Badel, "Non-linear losses study in strongly coupled piezoelectric device for broadband energy harvesting," Mech. Syst. Signal Process., vol. 165, p. 108370, Feb. 2022, doi: 10.1016/j.ymssp.2021.108370.

[7] A. Morel, A. Badel, R. Grézaud, P. Gasnier, G. Despesse, and G. Pillonnet, "Resistive and reactive loads' influences on highly coupled piezoelectric generators for wideband vibrations energy harvesting," J. Intell. Mater. Syst. Struct., vol. 30, no. 3, Art. no. 3, Feb. 2019, doi: 10.1177/1045389X18810802.

[8] B. Ahmed-Seddik, G. Despesse, S. Boisseau, and E. Defay, "Self-powered resonant frequency tuning for Piezoelectric Vibration Energy Harvesters," J. Phys. Conf. Ser., vol. 476, p. 012069, Dec. 2013, doi: 10.1088/1742-6596/476/1/012069. 